\title{
Configuration Analysis and Gene Modeling of Multi Knuckles Nimble Finger with Metamorphic Function
}

\author{
Xianzhi $\mathrm{Li}^{1, \mathrm{a}^{*} \text {, Xijun Yang }}{ }^{2, \mathrm{~b}}$,Siyu Chen ${ }^{3, \mathrm{c}}$ and Feng $\mathrm{Yu}^{4, \mathrm{~d}}$ \\ 1-4 Jiamusi University, Jiamusi, Heilongjiang, 154007, China \\ axz305@126.com, bYangxijun12345@126.com, '20862779@qq.com \\ *Corresponding author: Xianzhi Li, E-mail:Ixz305@126.com,TEL:+86-13512677079
}

Keywords: Metamorphic function, Multi knuckles nimble finger, Structure analysis, Correlation matrix, Genetic model.

Abstract. Put three knuckles nimble finger as the research object, the paper described its configurations by the application of topology and degree of freedom, expressed mechanism topology characteristics by association matrix based on the metamorphic mechanism theory, analyzed configuration transformation process of the metamorphic nimble finger with multi knuckles by using XOR logic operation method. This method can describe the configuration transformation process clearly, and can describe the changes of the number and type of motion pair. A genetic model of metamorphic mechanism was built based on the metamorphic elements, discussed configuration evolution and evolution of metamorphic nimble fingers with multi knuckles.

\section{Introduction}

The nimble finger has been used widely in various mechanical field, it can adapt to a variety of complex and delicate task. The paper described the configuration by the application of topology and degree of freedom based on the theory of metamorphic mechanism, expressed mechanism topology characteristics by association matrix, analyzed configuration transformation process of the metamorphic finger mechanism by using XOR logic operation method. A genetic model of metamorphic mechanism was built based on the metamorphic elements to discuss configuration evolution.

\section{The Metamorphic Mechanism Theory}

Metamorphic mechanism is a kind of mechanism with change of the functional, the topology and the degree of freedom. Because of its adaptability and reconstruction, metamorphic mechanism is especially suitable for different stages of different task requirements.

Configuration change is one of the most important characteristics of metamorphic mechanism, the configuration change is affected by different linkage and physical limits, it can be characterized by topological structure and degree of freedom. Dai J S and Zhang Q X introduced the concept of metamorphic mechanism to China, and used the method of matrix operation to analyze further the configuration transformation of some metamorphic mechanism examples [1]. Li D $\mathrm{L}$ proposed a simple algorithm on structure changing of metamorphic mechanisms and a kind of new synthetic method on metamorphic mechanism based on matrix operation [2].Zhang L P investigated the gene modeling theory of metamorphic mechanism, analyzed the configuration evolution, synthesized metamorphic mechanism based on gene modeling and the evolution $[3,4]$.

\section{Configuration Analysis of Three Knuckles Nimble Finger}

According to the configuration description method of literature [5], metamorphic processes were described by using correlation matrix with motion pair type and logic operation, the motion pair type 
is introduced to correlation matrix. Describe the configuration transformation process and all topological variation by using a logical XOR operation for correlation matrix.

\section{Configuration Analysis of Metamorphic Source Mechanism}

Mechanism agency brief is shown in Fig. 1(a), component 8 is nearly knuckle, component 7 is middle knuckle, member 6 is the far knuckle. There are extension springs between nearly knuckle and middle knuckle, between the middle knuckle and far knuckle to limit mechanism keep unchanged. Its topological graph is shown in Fig. 1(b), the variable member is expressed by point, motion pair is expressed by linear. Fig. 1 is source configuration of the nimble finger with three knuckles, namely the topological characteristics before nimble finger contact with holding clamp.

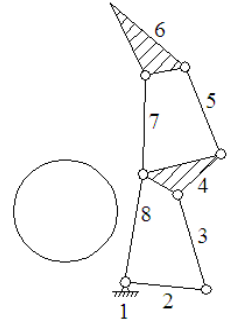

(a) Agency brief

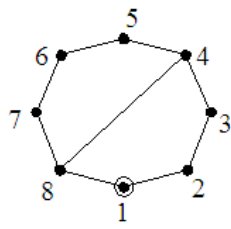

(b) Topological graph

Fig. 1 Metamorphic source mechanism

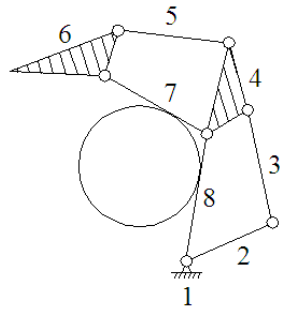

(a) Agency brief

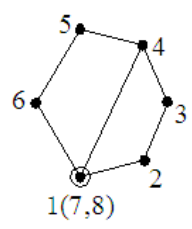

(b) Topological graph

Fig. 3 The second configuration

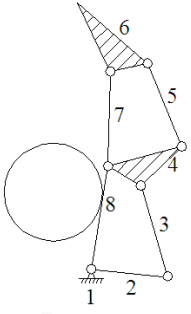

(a) Agency brief

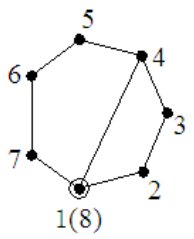

(b) Topological graph

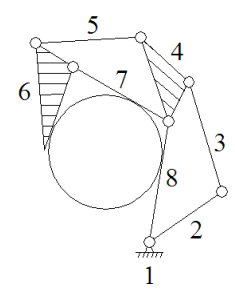

(a) Agency brief

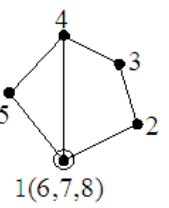

(b) Topological graph Fig. 4 The third configuration

The degree of freedom of mechanism can be calculated. The expression is:

$$
F=3 n-\left(2 p_{l}+p_{h}\right)=3 \times 7-2 \times 9=3
$$

The correlation matrix is used to express the characteristics of mechanism topology, the correlation matrix is:

$$
A^{0}=\left[\begin{array}{lllllllll}
R & R & R & R & R & R & R & R & R \\
1 & 0 & 0 & 0 & 0 & 0 & 0 & 0 & 1 \\
1 & 1 & 0 & 0 & 0 & 0 & 0 & 0 & 0 \\
0 & 1 & 1 & 0 & 0 & 0 & 0 & 0 & 0 \\
0 & 0 & 1 & 1 & 1 & 0 & 0 & 0 & 0 \\
0 & 0 & 0 & 1 & 0 & 1 & 0 & 0 & 0 \\
0 & 0 & 0 & 0 & 0 & 1 & 1 & 0 & 0 \\
0 & 0 & 0 & 0 & 0 & 0 & 1 & 1 & 0 \\
0 & 0 & 0 & 0 & 0 & 0 & 0 & 1 & 1
\end{array}\right]
$$

The above equations represent mechanism of nimble finger with three knuckles, correlation matrix topology is $9 \times 9$ matrix and the mechanism has 3 degrees of freedom.

\section{The First Configuration Analysis of Three Knuckles Nimble Finger}

When the nearly knuckle 8 move to contact the gripped objects gradually, gripped objects will have a reaction to the knuckle, restrict its movement, equivalent to the 8 member into frame. Topology structure of nimble finger will change, degree of freedom can change. The first configuration of nimble finger with three knuckles is shown in Fig. 2.

The degree of freedom of mechanism can be calculated. The expression is: 


$$
F=3 n-\left(2 p_{l}+p_{h}\right)=3 \times 6-2 \times 8=2
$$

Transformation matrix is:

$$
B_{1}^{\left(l_{1},-l_{8}\right)}=\left[\begin{array}{ccccccccc}
0 & 0 & 0 & 0 & 0 & 0 & 0 & 0 & 0 \\
0 & 0 & 0 & 0 & 0 & 0 & 0 & 1 & 1 \\
0 & 0 & 0 & 0 & 0 & 0 & 0 & 0 & 0 \\
0 & 0 & 0 & 0 & 0 & 0 & 0 & 0 & 0 \\
0 & 0 & 0 & 0 & 0 & 0 & 0 & 0 & 0 \\
0 & 0 & 0 & 0 & 0 & 0 & 0 & 0 & 0 \\
0 & 0 & 0 & 0 & 0 & 0 & 0 & 0 & 0 \\
0 & 0 & 0 & 0 & 0 & 0 & 0 & 0 & 0 \\
0 & 0 & 0 & 0 & 0 & 0 & 0 & 1 & 1
\end{array}\right]
$$

Its correlation matrix is:

$$
A^{1}=A^{0} \oplus B_{1}^{\left(l_{1},-l_{8}\right)}=\left[\begin{array}{ccccccccc}
R & R & R & R & R & R & R & R & R \\
1 & 0 & 0 & 0 & 0 & 0 & 0 & 1 & 0 \\
1 & 1 & 0 & 0 & 0 & 0 & 0 & 0 & 0 \\
0 & 1 & 1 & 0 & 0 & 0 & 0 & 0 & 0 \\
0 & 0 & 1 & 1 & 1 & 0 & 0 & 0 & 0 \\
0 & 0 & 0 & 1 & 0 & 1 & 0 & 0 & 0 \\
0 & 0 & 0 & 0 & 0 & 1 & 1 & 0 & 0 \\
0 & 0 & 0 & 0 & 0 & 0 & 1 & 1 & 0 \\
0 & 0 & 0 & 0 & 0 & 0 & 0 & 0 & 0
\end{array}\right]
$$

The three types represent the first configuration of nimble finger with three knuckles, which is nearly knuckle 8 contact the clamping, movable components of mechanism reduce, topology structure changes, degree of freedom reduced to 2 , respectively.

\section{The Second Configuration Analysis of Nimble Finger with Three Knuckles}

When the middle knuckle 7 turn to contact the clamping, nimble finger is in the second configuration, contact forced limit the motion of the component 7, 7 and 1 (8) were merged into the new frame in the topology. The mechanism topological structure and degree of freedom will change, is shown in Fig. 3.

The degree of freedom of mechanism can be calculated. The expression is:

$$
F=3 n-\left(2 p_{l}+p_{h}\right)=3 \times 5-2 \times 7=1
$$

Transformation matrix is:

$$
B_{2}^{\left(l_{1},-l_{7}\right)}=\left[\begin{array}{ccccccccc}
0 & 0 & 0 & 0 & 0 & 0 & 0 & 0 & 0 \\
0 & 0 & 0 & 0 & 0 & 0 & 1 & 1 & 0 \\
0 & 0 & 0 & 0 & 0 & 0 & 0 & 0 & 0 \\
0 & 0 & 0 & 0 & 0 & 0 & 0 & 0 & 0 \\
0 & 0 & 0 & 0 & 0 & 0 & 0 & 0 & 0 \\
0 & 0 & 0 & 0 & 0 & 0 & 0 & 0 & 0 \\
0 & 0 & 0 & 0 & 0 & 0 & 0 & 0 & 0 \\
0 & 0 & 0 & 0 & 0 & 0 & 1 & 1 & 0 \\
0 & 0 & 0 & 0 & 0 & 0 & 0 & 0 & 0
\end{array}\right]
$$

Its correlation matrix is:

$$
A^{2}=A^{1} \oplus B_{2}^{\left(l_{1},-l_{7}\right)}=\left[\begin{array}{ccccccccc}
R & R & R & R & R & R & R & R & R \\
1 & 0 & 0 & 0 & 0 & 0 & 1 & 0 & 0 \\
1 & 1 & 0 & 0 & 0 & 0 & 0 & 0 & 0 \\
0 & 1 & 1 & 0 & 0 & 0 & 0 & 0 & 0 \\
0 & 0 & 1 & 1 & 1 & 0 & 0 & 0 & 0 \\
0 & 0 & 0 & 1 & 0 & 1 & 0 & 0 & 0 \\
0 & 0 & 0 & 0 & 0 & 1 & 1 & 0 & 0 \\
0 & 0 & 0 & 0 & 0 & 0 & 0 & 0 & 0 \\
0 & 0 & 0 & 0 & 0 & 0 & 0 & 0 & 0
\end{array}\right]
$$




\section{The Third Configuration Analysis of Nimble Finger with Three Knuckles}

When the far knuckle 6 rotates to contact the clamping, nimble finger is in the third configuration, contact force limits movement of far knuckle 6 , knuckle 6 and $1(7,8)$ were merged into the new frame in the topology. The mechanism topological structure and degree of freedom will change, is shown in Fig. 4.

The degree of freedom of mechanism can be calculated. The expression is:

$$
F=3 n-\left(2 p_{l}+p_{h}\right)=3 \times 4-2 \times 6=0
$$

Transformation matrix is:

$$
B_{3}^{\left(l_{1},-l_{6}\right)}=\left[\begin{array}{ccccccccc}
0 & 0 & 0 & 0 & 0 & 0 & 0 & 0 & 0 \\
0 & 0 & 0 & 0 & 0 & 1 & 1 & 0 & 0 \\
0 & 0 & 0 & 0 & 0 & 0 & 0 & 0 & 0 \\
0 & 0 & 0 & 0 & 0 & 0 & 0 & 0 & 0 \\
0 & 0 & 0 & 0 & 0 & 0 & 0 & 0 & 0 \\
0 & 0 & 0 & 0 & 0 & 1 & 1 & 0 & 0 \\
0 & 0 & 0 & 0 & 0 & 0 & 0 & 0 & 0 \\
0 & 0 & 0 & 0 & 0 & 0 & 0 & 0 & 0 \\
0 & 0 & 0 & 0 & 0 & 0 & 0 & 0 & 0
\end{array}\right]
$$

Its correlation matrix is:

$$
A^{3}=A^{2} \oplus B_{3}^{\left(l_{1},-l_{6}\right)}=\left[\begin{array}{ccccccccc}
R & R & R & R & R & R & R & R & R \\
1 & 0 & 0 & 0 & 0 & 1 & 0 & 0 & 0 \\
1 & 1 & 0 & 0 & 0 & 0 & 0 & 0 & 0 \\
0 & 1 & 1 & 0 & 0 & 0 & 0 & 0 & 0 \\
0 & 0 & 1 & 1 & 1 & 0 & 0 & 0 & 0 \\
0 & 0 & 0 & 1 & 0 & 1 & 0 & 0 & 0 \\
0 & 0 & 0 & 0 & 0 & 0 & 0 & 0 & 0 \\
0 & 0 & 0 & 0 & 0 & 0 & 0 & 0 & 0 \\
0 & 0 & 0 & 0 & 0 & 0 & 0 & 0 & 0
\end{array}\right]
$$

The topological graph has 0 degree of freedom, the finger can complete stable grasping function, achieve the goal of design. Based on the analysis of the above configuration, in the process of nimble finger contacting clamping, the finger mechanism experienced four configuration transformations, the degree of freedom changed from 3 to 2, 1, 0 , so that the finger in a control can drive movement of three knuckles , and complete stable grasping function.

This can explain fundamentally the metamorphic principle of capture process of nimble finger with multi knuckles, and provide a theoretical basis for the mechanism design and control planning of nimble finger.

\section{Gene Modeling of Finger with Three Knuckles}

\section{Gene Modeling of Metamorphic Source Mechanism}

According to the theory of literatures $[3,4]$, metamorphic mechanism has multiple configurations corresponding different working stages in the work cycle, it can be described by evolution, gene cell and cell combined evolving metamorphic elements with metamorphic characteristics. Gene modeling expression of metamorphic mechanism is .

$$
{ }^{s} M=\left[a_{\mathrm{i}} ; A_{\mathrm{i}} ; a_{\mathrm{f}}\right]
$$

In the formula, $a_{\mathrm{i}}$ is dynamic gene, $A_{\mathrm{i}}$ is gene cell, $a_{\mathrm{f}}$ is fixed gene .Genetic structure within the organization is shown in Fig. 5. 


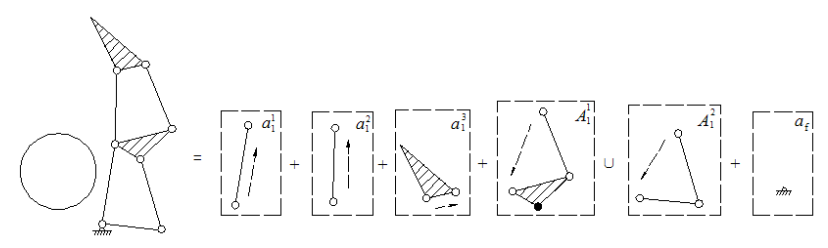

Fig. 5 The metamorphic unit structure of metamorphic source mechanism

\section{Analysis of Metamorphic Evolution Process}

From the above analysis, there are three sub working stages, corresponding to the three different topological structures. Their expressions of gene model structures are:

$$
\begin{aligned}
{ }^{1} M & =\left[a_{1}^{1}, a_{1}^{2} ; A_{1}^{1}, A_{1}^{2} ; a_{f}\right] \\
{ }^{2} M & =\left[a_{1}^{2} ; A_{1}^{1}, A_{1}^{2} ; a_{f}\right] \\
{ }^{3} M & =\left[a_{f}\right]
\end{aligned}
$$

The internal gene structure can be decomposed, it is shown in Fig. 6.

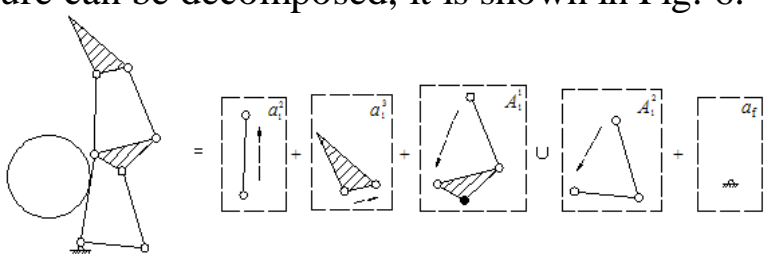

(a) ' $M$ gene model structure

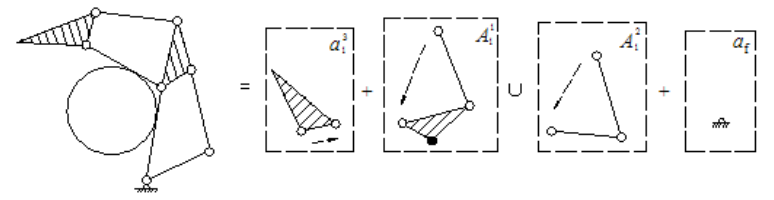

(b) ${ }^{2} M$ gene model structure

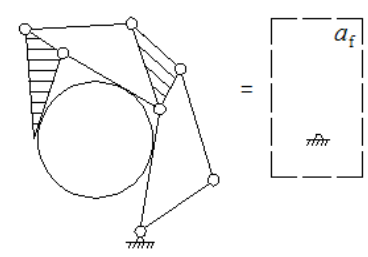

(c) ${ }^{3} M$ gene model structure

Fig. 6 Gene model structure of mechanism working stages

The working stages mechanism of ${ }^{1} M,{ }^{2} M$ and ${ }^{3} M$ can evolve into metamorphic source mechanism. The evolution expression is:

$$
{ }^{s} M={ }^{1} M \mathrm{U}^{2} M \mathrm{U}^{3} M=\left[a_{1}^{1}, a_{1}^{2} ; A_{1}^{1}, A_{1}^{2} ; a_{f}\right] \mathrm{U}\left[a_{1}^{2} ; A_{1}^{1}, A_{1}^{2} ; a_{f}\right] \mathrm{U}\left[a_{f}\right]
$$

The mechanism evolution expression of working stages mechanism of ${ }^{1} M$ and ${ }^{2} M$ is:

$$
{ }^{1} M \mathrm{U}^{2} M=\left[a_{1}^{1}, a_{1}^{2} ; A_{1}^{1}, A_{1}^{2} ; a_{f}\right] \cup\left[a_{1}^{2} ; A_{1}^{1}, A_{1}^{2} ; a_{f}\right]
$$

In particular, there is same active cell in the configuration 1 and configuration 2, so one configuration of the mechanism need to transfer a dynamic gene in order to find the most simple metamorphic source kinematic chain. For ${ }^{2} M$

$$
{ }^{2} M=\left[a_{1}^{2} ; A_{1}^{1}, A_{1}^{2} ; a_{f}\right]=\left[a_{1}^{3} ; a_{1}^{2} \cup A_{1}^{1} \backslash a_{1}^{3}, a_{1}^{2} \cup A_{1}^{2} \backslash a_{1}^{3} ; a_{f}\right]
$$

Then the type (17) can be written in the following form.

$$
\begin{aligned}
& { }^{1} M \cup^{2} M=\left[a_{1}^{1}, a_{1}^{2} ; A_{1}^{1}, A_{1}^{2} ; a_{f}\right] \cup\left[a_{1}^{2} ; A_{1}^{1}, A_{1}^{2} ; a_{f}\right]= \\
& {\left[a_{1}^{1}, a_{1}^{2} ; A_{1}^{1}, A_{1}^{2} ; a_{f}\right] \cup\left[a_{1}^{3} ; a_{1}^{2} \cup A_{1}^{1} \backslash a_{1}^{3}, a_{1}^{2} \cup A_{1}^{2} \backslash a_{1}^{3} ; a_{f}\right]=} \\
& {\left[\left(a_{1}^{1}+a_{1}^{2}+a_{1}^{3}\right) ;\left(A_{1}^{1} \cup A_{1}^{2} \cup a_{1}^{2} \cup\left(A_{1}^{1} \backslash a_{1}^{3}\right) \cup a_{1}^{2} \cup\left(A_{1}^{2} \backslash a_{1}^{3}\right)\right) ;\left(a_{\mathrm{f}} \cup a_{\mathrm{f}}\right)\right]=} \\
& {\left[a_{1}^{1}, a_{1}^{2}, a_{1}^{3} ; A_{1}^{1}, A_{1}^{2} ; a_{f}\right]}
\end{aligned}
$$


The convergent evolution result of type (19) and ${ }^{3} M$ is:

$$
{ }^{s} M={ }^{1} M \mathrm{U}^{2} M \mathrm{U}^{3} M=\left[a_{1}^{1}, a_{1}^{2}, a_{1}^{3} ; A_{1}^{1}, A_{1}^{2} ; a_{f}\right]
$$

The evolution process is shown in Fig. 7.

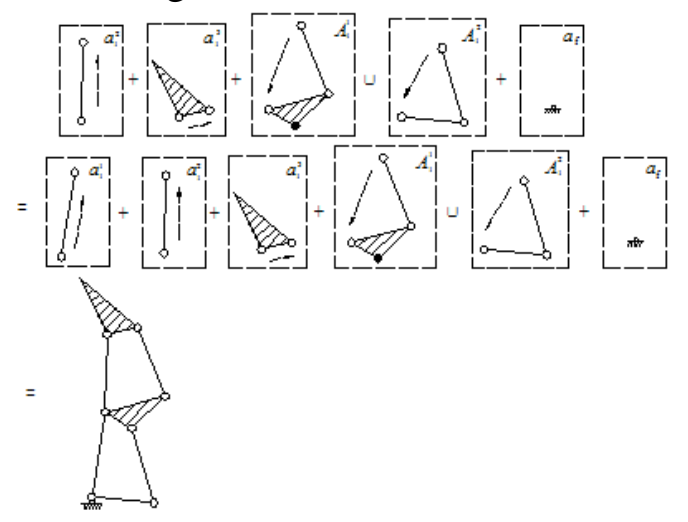

Fig. 7 Evolution process schematic diagram of metamorphic source mechanism

\section{Conclusions}

The metamorphic theory is applied to the design of nimble finger mechanism. The correlation matrix and topological diagram are used to describe the topological structure of finger mechanism configuration. The paper analyzed configuration change process of metamorphic finger mechanism by using the arithmetic logic XOR, established every configuration gene model of the finger mechanism according to the gene modeling theory, got gene expression of source mechanism model through convergent evolution of each stage configuration.

\section{Acknowledgements}

This work was supported by Provincial Education Department Foundation of Heilongjiang, China(No. 12541785) and college students innovative training program of Heilongjiang Province(201510222001).

\section{References:}

[1]Dai J S, Zhang Q X(2000). Metamorphic mechanisms and their configuration models. Chinese Journal of Mechanical Engineering, ,13(3):212-218.

[2]Li D L, Zhang Q X, Dai J S(2002). Synthesis of a metamorphic mechanism based on configuration analysis.Professional Engineering Publishing Limited Bury St Edmunds and London, 563-575.

[3]Zhang L P, Wang D L, Dai J S(2008). Genetic modeling theory and configuration evolution analysis of metamorphic mechanism. Chinese Journal of Mechanical Engineering,44 (12): 49-56. In Chinese.

[4]Zhang L P, Wang D L, Dai J S(2008). Biological modeling and evolution based synthesis of metamorphic mechanisms. ASME Journal of Mechanical Design,130: 072303-1-072303-11. In Chinese.

[5]Chang B Y, Jin G G, Dai J S(2011). Correlation matrix method to describe the structure changing of metamorphic mechanisms. Mechanical Science and Technology,30(3):507-512. In Chinese. 